\title{
Tensor invariants of generalized Kenmotsu manifolds
}

\author{
Ali Abdul Al Majeed Shihab ${ }^{1}$ and Aligadzhi Rustanov ${ }^{2, *}$ \\ ${ }^{1}$ Mathematics college of Education for Women - University of Tikrit, Iraq Salahaddin-Tikrit, p.o. Box \\ 42, Tikrit. Iraq \\ ${ }^{2}$ Moscow State University of Civil Engineering, Yaroslavskoe shosse, 26, 129337, Moscow, Russia
}

\begin{abstract}
In this paper, we study the properties of generalized Kenmotsu manifolds, consider the second-order differential geometric invariants of the Riemannian curvature tensor of generalized Kenmotsu manifolds (by the symmetry properties of the Riemannian geometry tensor). The concept of a tensor spectrum is introduced. Nine invariants are singled out and the geometric meaning of these invariants turning to zero are investigated. The identities characterizing the selected classes are singled out. Also, 9 classes of generalized Kenmotsu manifolds are distinguished, the local structure of 8 classes from the selected ones is obtained.
\end{abstract}

\section{Introduction}

Let $M$ be a connected smooth manifold of dimensions $(2 n+1), C^{\infty}(M)$ the algebra of smooth functions on $M, X(M)-C^{\infty}$-module of smooth vector fields on the manifold $M, \mathrm{~d}$ an outer derivation operator. If a Riemannian metric $\langle\cdot, \cdot\rangle$ is given on $M$, then the corresponding Riemannian connection will be marked by the symbol $\nabla$. All manifolds, tensor fields (tensors), etc. objects are assumed to be smooth of class $C^{\infty}$.

Differential 1-forms of maximum rank on an odd-dimensional Riemannian manifold generate a special differential-geometric structure, called a contact metric structure, which naturally generalizes to a so-called almost contact metric structure.

A lot of works are devoted to the study of almost contact metric structures. We have chosen for a study a rather interesting class of almost contact metric structures, called the class of generalized Kenmotsu manifolds.

Generalized Kenmotsu manifolds were first introduced as approximately trans-Sasakian manifolds in the study [1]. In this paper discloses relations in this class of manifolds. In addition, some new classes of almost contact metric manifolds are introduced and the inclusion relations of these classes with generalized Kenmotsu manifolds are presented. It is shown that normal generalized Kenmotsu varieties are Kenmotsu varieties.

Further insight into the geometry of generalized Kenmotsu manifolds is carried out in the research paper by S.V. Umnova [2], where the definition of generalized (in short, $G K$-) Kenmotsu manifolds is given. In work [2] Umnova S.V. distinguishes two subclasses of generalized Kenmotsu varieties, called special generalized Kenmotsu varieties (for short,

\footnotetext{
*Corresponding author: aligadzhi@yandex.ru
} 
$S G K$-) of I and II types. It is proved that generalized Kenmotsu manifolds of constant curvature are Kenmotsu manifolds of constant curvature -1 . In addition, it is proved that the class of SGK-manifolds of the second kind coincides with the class of almost contact metric manifolds obtained from the most exact cosymplectic manifolds by a canonical transformation of the most exact cosymplectic structure, and the local structure of these manifolds of constant curvature is also given.

Papers [3 - 8] dwell on semi-invariant, completely umbilical semi-invariant, pseudo-slant submanifolds of generalized Kenmotsu manifolds, and also on semi-invariant submanifolds of generalized Kenmotsu manifolds with semi-symmetric, canonical semi-symmetric, quarter semi-symmetric non-metric connection. In these and other studies, generalized Kenmotsu manifolds are called nearly Kenmotsu manifolds. We call them generalized Kenmotsu varieties. Thus, in the paper [9], this class of manifolds is called the nearly Kenmotsu class of manifolds. The authors prove that a second-order symmetric closed recurrent tensor, whose recurrence covector annihilates the characteristic vector $\xi$, is a multiple of the metric tensor $g$. In addition, the authors consider $\Phi$-approximately Kenmotsu manifolds. It is proved that $\Phi$-recurrent approximately Kenmotsu manifolds are Einstein manifolds, and locally $\Phi$-recurrent approximately Kenmotsu manifolds are manifolds of constant curvature -1 .

In the paper [10], $S G K$ - manifolds of the second type are called nearly Kenmotsu manifolds. In the paper [11], $G K$-manifolds are called nearly Kenmotsu manifolds. The author proves that the characteristic vector of an approximately Kenmotsu structure is not a killing vector. It is proved that an approximately normal Kenmotsu structure is a Kenmotsu structure. It is also shown that quasi-Kenmotsu and nearly Kenmotsu structures intersect in Kenmotsu structures.

In the paper [12], identities of curvature of $S G K$-manifolds of the second type were obtained, and on their basis some subclasses of $S G K$-manifolds of the second type were distinguished. The form of the tensor of the holomorphic sectional curvature of $S G K$ manifolds of the second type of class $R_{4}$ is obtained and it is proved that $S G K$-manifolds of the second type of class $R_{5}$ are Kenmotsu manifolds. In the paper [13], contact analogs of the Gray identities for generalized Kenmotsu varieties are considered. The local structure of $G K$ manifolds satisfying contact analogs of Gray identities is obtained. Moreover, it was proved that almost $C(\lambda)$-manifolds that are $G K$-manifolds are Kenmotsu manifolds.

In the paper [14], some structural and nearly Kenmotsu curvature identities were obtained for manifolds. It is shown that a nearly normal Kenmotsu structure is a Kenmotsu structure. It is proved that, nearly Kenmotsu, a manifold is locally isometric to the skew product of the real line and a nearly Kähler manifold. In addition, it is shown that there are no nearly Kenmotsu hypersurfaces of nearly Kähler manifolds.

The work [15] is devoted to the study of an nearly Kenmotsu Einstein manifold satisfying the conditions $R(X, Y) \cdot P=0$ and $R(X, Y) \cdot N=0$, where $P$ is the projective curvature tensor and $N$ is the conharmonic curvature tensor. It is proved that nearly Kenmotsu of an Einstein manifold satisfying these conditions is locally isometric to the hyperbolic space $H^{n}(-1)$.

In the paper [16], it is proved that a contact distorted $C R$-skewed product does not exist in almost Kenmotsu manifolds. Provided it is true, a relation is given for the squared norm of the second fundamental immersion form of the skewed product.

In the paper [17], the authors study the geometry and topology of $\eta$-Ricci solitons satisfying the Ricci-semisymmetry condition, the $S \cdot R=0$ condition and, finally, the Einstein-semisymmetry condition on nearly Kenmotsu manifolds.

In the paper [18], the authors introduced a contact analog of type constancy and obtained a complete classification of generalized Kenmotsu manifolds of constant type. 
In the paper [19], it is proved that a $G K$-manifold of dimension other than 5 is an $S G K$ manifold of the second type. Consequently, we get that a $G K$-manifold, which is an $S G K$ manifold of the first type, has dimension 5.

Paper [20] investigates the integrability properties of generalized Kenmotsu manifolds. The first, second and third fundamental identities of $G K$-structures are defined. In this paper, we investigate $G K$-manifolds, the first fundamental distribution of which is completely integrable. It is shown that an almost Hermitian structure induced on integral manifolds of maximum dimension of the first distribution of a $G K$-manifold is nearly Kähler. The local structure of a $G K$-manifold with a closed contact form is obtained, expressions for the first and second structure tensors are given. The geometric meaning of the vanishing of the tensors $N^{(1)}, N^{(2)}, N^{(3)}, N^{(4)}$ is investigated. The local structure of an integrable and normal $G K$ structure is obtained. It is proved that the characteristic vector of the $G K$-structure is not a Killing vector.

From the above historical survey of works devoted to generalized Kenmotsu varieties, it is clear, that the interest to such studies does not fade away, though grows.

In this paper, we continue the study of generalized Kenmotsu manifolds and investigate second-order differential geometric invariants of the Riemannian curvature tensor of generalized Kenmotsu manifolds.

This paper is structured as follows. In Section 1, we give preliminary information needed in the further presentation: we construct the space of the associated $G$-structure of an almost contact metric manifold; we write down the first group of structural equations of an almost contact metric manifold on the space of the associated $G$-structure; we introduce structural tensors of an almost contact metric structure and present their properties.

In Section 2, we give a definition of generalized Kenmotsu manifolds, give a complete group of structure equations, and give five fundamental identities of generalized Kenmotsu manifolds. In Section 3, we present the components of the Riemann-Christoffel tensor on the space of the associated $G$-structure, and introduce the concept of the tensor spectrum. Nine tensor invariants of generalized Kenmotsu manifolds are distinguished, identities characterizing these invariants are obtained, and 9 classes of generalized Kenmotsu manifolds are distinguished. In addition, we obtain a local characterization of 8 of the selected classes.

\section{Preliminary information}

Let $M$ be a smooth manifold of dimension $2 n+1$.

Definition 1.1 [21]. An almost contact structure on a manifold $M$ is a triple $(\eta, \xi, \Phi)$ of tensor fields on this manifold, where $\eta$ is a differential 1 -form called the contact form of the structure, $\xi$ is a vector field called characteristic, $\Phi$ is an endomorphism of the module $\mathcal{X}(M)$ called structural endomorphism. Herewith

$$
\text { 1) } \eta(\xi)=1 \text {; 2) } \eta \circ \Phi=0 \text {; 3) } \Phi(\xi)=0 \text {; 4) } \Phi^{2}=-i d+\eta \otimes \xi \text {. }
$$

If, in addition, a Riemannian structure $g=\langle\cdot, \cdot\rangle$ is fixed on $M$ such that

$$
\langle\Phi X, \Phi Y\rangle=\langle\mathrm{X}, \mathrm{Y}\rangle-\eta(X) \eta(Y), X, Y \in \mathcal{X}(M),
$$

the quadruple $(\eta, \xi, \Phi, g=\langle\because\rangle$,$) is called an almost contact metric (in short, AC-) structure.$ A manifold on which an almost contact (metric) structure is fixed is called an almost contact (metric (in short, $A C$-)) manifold.

The skew-symmetric tensor $\Omega(X, Y)=\langle X, \Phi Y\rangle, X, Y \in \mathcal{X}(M)$ is called the fundamental form of the AC-structure [21]. 
Let $(\eta, \xi, \Phi, g)$ be an almost contact metric structure on the manifold $M^{2 n+1}$. In the module $X(M)$, two mutually complementary projectors $m=\eta \otimes \xi$ and $\ell=i d-m=$ $-\Phi^{2}$ are defined internally; thus, $\mathcal{X}(M)=\mathcal{L} \oplus \mathcal{M}$, where $\mathcal{L}=\operatorname{Im}(\Phi)=$ ker $\eta$ is the socalled contact (or first fundamental) distribution, $\operatorname{dim} \mathcal{L}=2 n, \mathcal{M}=\operatorname{Imm}=\operatorname{ker}(\Phi)=$ $L(\xi)$ - the linear hull of the structure vector or the so-called second fundamental distribution (moreover, $\ell$ and $m$ are projectors onto the submodules $\mathcal{L}, \mathcal{M}$, respectively) [21]. Obviously, the distributions $\mathcal{L}$ and $\mathcal{M}$ are invariant with respect to $\Phi$ and mutually orthogonal. It is also obvious that $\widetilde{\Phi}^{2}=-i d,\langle\widetilde{\Phi} X, \widetilde{\Phi} Y\rangle=\langle X, Y\rangle, X, Y \in \mathcal{X}(M)$, where $\widetilde{\Phi}=\Phi \mid \mathcal{L}$. Therefore, $\left\{\widetilde{\Phi}_{p}, g_{p} \mid \mathcal{L}\right\}$ is a Hermitian structure on the space $\mathcal{L}_{p}$.

The complexification $\chi(M)^{C}$ of the module $\chi(M)$ decomposes into a direct sum $\chi(M)^{\mathrm{C}}=D_{\Phi}^{\sqrt{-1}} \oplus D_{\Phi}^{-\sqrt{-1}} \oplus D_{\Phi}^{0}$ proper subspaces of the structural endomorphism $\Phi$ corresponding to eigenvalues $\sqrt{-1}, \sqrt{-1}$ and 0 , respectively. Moreover, the projectors onto the terms of this direct sum are, respectively, endomorphisms [21] $\pi=\sigma \circ \ell=$ $-\frac{1}{2}\left(\Phi^{2}+\sqrt{-1} \Phi\right), \bar{\pi}=\bar{\sigma} \circ \ell=\frac{1}{2}\left(-\Phi^{2}+\sqrt{-1} \Phi\right), m=i d+\Phi^{2}, \quad$ where $\sigma=\frac{1}{2}(i d-$ $\sqrt{-1} \Phi), \bar{\sigma}=\frac{1}{2}(i d+\sqrt{-1} \Phi)$.

Displayed values $\sigma_{p}: \mathcal{L}_{p} \rightarrow\left(D_{\Phi}^{\sqrt{-1}}\right)_{p}$ and $\sigma \bar{\sigma}_{p}: \mathcal{L}_{p} \rightarrow\left(D_{\Phi}^{-\sqrt{-1}}\right)_{p}$ are isomorphisms and antiisomorphisms, respectively, of Hermitian spaces. Therefore, to each point $p \in M^{2 n+1}$ one can attach a family of frames of the space $T_{p}(M)^{C}$ of the form $\left(p, \varepsilon_{0}, \varepsilon_{1}, \ldots, \varepsilon_{n}, \varepsilon_{\hat{1}}, \ldots, \varepsilon_{\hat{n}}\right)$, where $\varepsilon_{a}=\sqrt{2} \sigma_{p}\left(e_{a}\right), \varepsilon_{\hat{a}}=\sqrt{2} \bar{\sigma}_{p}\left(e_{a}\right), \varepsilon_{0}=\xi_{p}$; where $\left\{e_{a}\right\}$ is an orthonormal basis of the Hermitian space $\mathcal{L}_{p}$. Such a frame is called an $A$-frame [21]. It is easy to see that the matrices of the components of the tensors $\Phi_{p}$ and $g_{p}$ in the $A$-frame have the form, respectively:

$$
\left(\Phi_{j}^{i}\right)=\left(\begin{array}{ccc}
0 & 0 & 0 \\
0 & \sqrt{-1} I_{n} & 0 \\
0 & 0 & -\sqrt{-1} I_{n}
\end{array}\right),\left(g_{i j}\right)=\left(\begin{array}{ccc}
1 & 0 & 0 \\
0 & 0 & I_{n} \\
0 & I_{n} & 0
\end{array}\right),
$$

where $I_{n}$ is the identity matrix of order $n$. It is well known [21] that the set of such frames defines a $G$-structure on $M$ with the structure group $\{1\} \times U(n)$ represented by matrices of the form $\left(\begin{array}{ccc}1 & 0 & 0 \\ 0 & A & 0 \\ 0 & 0 & A\end{array}\right)$, where $A \in U(n)$. This $G$-structure is called adjoint [21].

Let $\left(M^{2 n+1}, \Phi, \xi, \eta, g=\langle\cdot\rangle,\right)$ be an almost contact metric manifold. Let us agree that throughout the entire work, unless otherwise stated, the indices $i, j, k, l, \ldots$ run through the values from 1 to $2 n$, the indices $a, b, c, d, \ldots$ are values from 1 to $n$, and put $\hat{a}=a+n, \hat{a}=$ $a, \hat{0}=0$. Let $(U, \varphi)$ be a local map on a manifold $M$. According to the Basic Theorem of tensor analysis [22, p. 243], the assignment of a structural endomorphism $\Phi$ and a Riemannian structure $g=\langle\cdot, \cdot\rangle$ on a manifold $M$ induces the assignment of a bundle on the total space $B M$ frames over $M$ of a system of functions $\left\{\Phi_{j}^{i}\right\},\left\{g_{i j}\right\}$ satisfying in the coordinate neighborhood $W=\pi^{-1}(U) \subset B M$ a system of differential equations of the form

$$
d \Phi_{j}^{i}+\Phi_{j}^{k} \theta_{k}^{i}-\Phi_{k}^{i} \theta_{j}^{k}=\Phi_{j, k}^{i} \omega^{k}, d g_{i j}-g_{k j} \theta_{i}^{k}-g_{i k} \theta_{j}^{k}=g_{i j, k} \omega^{k},
$$

where $\left\{\omega^{i}\right\},\left\{\theta_{j}^{i}\right\}$ are the components of the displacement forms and the Riemannian connection $\nabla$, respectively, $\Phi_{j, k}^{i}, g_{i j, k}$ are the components of the covariant differential of the tensors $\Phi$ and $g$ in this connection, respectively. Moreover, by the definition of the Riemannian connection, $\nabla g=0$ and, therefore,

$$
g_{i j, k}=0 \text {. }
$$



22]

Relations (1.4) on the space of the associated $G$-structure can be written in the form [21,

$$
\begin{gathered}
\Phi_{b, k}^{a}=0, \Phi_{\hat{b}, k}^{\hat{a}}=0, \Phi_{0, k}^{0}=0, \\
\theta_{\hat{b}}^{a}=\frac{\sqrt{-1}}{2} \Phi_{\hat{b}, k}^{a} \omega^{k}, \theta_{b}^{\hat{a}}=-\frac{\sqrt{-1}}{2} \Phi_{b, k}^{\hat{a}} \omega^{k}, \\
\theta_{0}^{a}=\sqrt{-1} \Phi_{0, k}^{a} \omega^{k}, \theta_{0}^{\hat{a}}=-\sqrt{-1} \Phi_{0, k}^{\hat{a}} \omega^{k}, \\
\theta_{a}^{0}=-\sqrt{-1} \Phi_{a, k}^{0} \omega^{k}, \theta_{\hat{a}}^{0}=\sqrt{-1} \Phi_{\hat{a}, k}^{0} \omega^{k}, \\
\theta_{j}^{i}+\theta_{\hat{\jmath}}^{\hat{\imath}}=0, \theta_{0}^{0}=0 .
\end{gathered}
$$

In addition, note that, since the corresponding forms and tensors are real, $\overline{\omega^{\imath}}=\omega^{\hat{\imath}}, \overline{\theta_{\jmath}^{\imath}}=$ $\theta_{\hat{\jmath}}^{\hat{\imath}}, \overline{\nabla \Phi_{J, k}^{l}}=\nabla \Phi_{\hat{\jmath}, \hat{k}}^{\hat{\imath}}$, where $t \rightarrow \bar{t}$ is the operator of complex conjugation.

The first group of structural equations of Riemannian connection $d \omega^{i}=-\theta_{j}^{i} \wedge \omega^{j}$, on the space of the associated $G$-structure of an almost contact metric manifold, can be written in the following form, called the first group of structure equations for an almost contact metric manifold $[21,22]$ :

$$
\begin{aligned}
d \omega & =C_{a b} \omega^{a} \wedge \omega^{b}+C^{a b} \omega_{a} \wedge \omega_{b}+C_{a}^{b} \omega^{a} \wedge \omega_{b}+C_{a} \omega \wedge \omega^{a}+C^{a} \omega \wedge \omega_{a} \\
d \omega^{a} & =-\theta_{b}^{a} \wedge \omega^{b}+B^{a b}{ }_{c} \omega^{c} \wedge \omega_{b}+B^{a b c} \omega_{b} \wedge \omega_{c}+B^{a b} \omega \wedge \omega_{b}+B^{a}{ }_{b} \omega \wedge \omega^{b}(1.7) \\
d \omega_{a} & =\theta_{a}^{b} \wedge \omega_{b}+B_{a b}{ }^{c} \omega_{c} \wedge \omega^{b}+B_{a b c} \omega^{b} \wedge \omega^{c}+B_{a b} \omega \wedge \omega^{b}+B_{a}{ }^{b} \omega \wedge \omega_{b},
\end{aligned}
$$

where $\omega=\omega^{0}=\pi^{*}(\eta) ; \pi$ is the natural projection of the space of the adjoint $G$-structure onto the manifold $M, \omega_{i}=g_{i j} \omega^{i}$,

$$
\begin{gathered}
B^{a b}{ }_{c}=-\frac{\sqrt{-1}}{2} \Phi_{\hat{b}, c}^{a}, B_{a b}{ }^{c}=\frac{\sqrt{-1}}{2} \Phi_{b, \hat{c}}^{\hat{a}}, B^{a b c}=\frac{\sqrt{-1}}{2} \Phi_{[\hat{b}, \hat{c}]}^{a}, \\
B_{a b c}=-\frac{\sqrt{-1}}{2} \Phi_{[b, c]}^{a}, B^{a}{ }_{b}=\sqrt{-1} \Phi_{0, b}^{a}, B_{a}{ }^{b}=-\sqrt{-1} \Phi_{0, \hat{b}}^{\hat{a}}, \\
B^{a b}=\sqrt{-1}\left(\Phi_{0, \hat{b}}^{a}-\frac{1}{2} \Phi_{\hat{b}, 0}^{a}\right), B_{a b}=-\sqrt{-1}\left(\Phi_{0, b}^{\hat{a}}-\frac{1}{2} \Phi_{b, 0}^{\hat{a}}\right), \\
C^{a b}=\sqrt{-1} \Phi_{[\hat{a}, \hat{b}]}^{0}, C_{a b}=-\sqrt{-1} \Phi_{[a, b]}^{0}, C_{a}^{b}=B_{a}^{b}{ }_{a}-B_{a}{ }^{b}=-\sqrt{-1}\left(\Phi_{\hat{b}, a}^{0}+\Phi_{a, \hat{b}}^{0}\right), \\
C^{a}=-\sqrt{-1} \Phi_{\hat{a}, 0}^{0}, C_{a}=\sqrt{-1} \Phi_{a, 0}^{0} .
\end{gathered}
$$

We introduce the notation [20]

$$
C^{a b c}=\frac{\sqrt{-1}}{2} \Phi_{\hat{b}, \hat{c}}^{a}, C_{a b c}=-\frac{\sqrt{-1}}{2} \Phi_{b, c}^{\hat{a}}, F^{a b}=\sqrt{-1} \Phi_{\hat{a}, \hat{b}}^{0}, F_{a b}=-\sqrt{-1} \Phi_{a, b}^{0} .
$$

Consider the following families of functions on the space of the associated $G$-structure [22]:

1) $B=\left\{B_{j k}^{i}\right\} ; B^{a}{ }_{\hat{b} c}=B^{a b}{ }_{c}, B^{\hat{a}}{ }_{b \hat{c}}=B_{a b}{ }^{c}$; all other components of family $B$ are zero;

2) $C=\left\{C^{i}{ }_{j k}\right\} ; C^{a}{ }_{\hat{b} \hat{c}}=C^{a b c} ; C^{\hat{a}}{ }_{b c}=C_{a b c}$; all other components of the family $C$ are zero;

3) $D=\left\{D_{j}^{i}\right\} ; D^{a}{ }_{\hat{b}}=B^{a b} ; D^{\hat{a}}{ }_{b}=B_{a b}$; all other components of the family $D$ are zero;

4) $E=\left\{E^{i}{ }_{j}\right\} ; E^{a}{ }_{b}=B^{a}{ }_{b} ; E^{\hat{a}_{\hat{b}}}=B_{a}{ }^{b}$; all other components of the family $E$ are zero;

5) $F=\left\{F_{j}^{i}\right\} ; F^{a}{ }_{\hat{b}}=F^{a b} ; F^{\hat{a}}{ }_{b}=F_{a b}$; all other components of the family $F$ are zero;

6) $G=\left\{G^{i}\right\} ; G^{a}=C^{a}$; $G^{a}=C_{a}$; all other components of the family $G$ are zero. 
These systems of functions define tensors of the corresponding types on the manifold $M$, which are called the first, second, ..., sixth structural tensors of the $A C$-structure, respectively. The following takes place

Proposition 1.1 [22]. Structural tensors of the $A C$-structure have the following properties:

1) $\Phi \circ B(X, Y)=-B(\Phi X, Y)=B(X, \Phi Y)$;

2) $\langle\langle B(X, Y), Z\rangle\rangle+\overline{\langle\langle Y, B(X, Z)\rangle\rangle}=0$;

3) $\Phi \circ C(X, Y)=-C(\Phi X, Y)=-C(X, \Phi Y)$;

4) $\langle\langle C(X, Y), Z\rangle\rangle+\overline{\langle\langle Y, C(X, Z)\rangle\rangle}=0$;

5) $\Phi \circ D=-D \circ \Phi$;

6) $\Phi \circ E=E \circ \Phi$;

7) $\Phi \circ F=-F \circ \Phi$;

8) $G \in \mathcal{L}$; где $\langle\langle X, Y\rangle\rangle=\langle X, Y\rangle+\sqrt{-1}\langle X, \Phi Y\rangle,(X, Y, Z \in X(M))$.

\section{Generalized Kenmotsu manifolds}

Let $\left(M^{2 n+1}, \Phi, \xi, \eta, g=\langle\cdot\rangle,\right)$ be an almost contact metric manifold.

Definition 2.1 ([2], [9], [12]). The class of almost contact metric manifolds characterized by the identity

$$
\nabla_{X}(\Phi) Y+\nabla_{Y}(\Phi) X=-\eta(Y) \Phi X-\eta(X) \Phi Y ; X, Y \in \mathcal{X}(M)
$$

are called generalized Kenmotsu manifolds (in short, GK-manifolds).

Note that in print this class of manifolds is called the nearly Kenmotsu class of manifolds ([3 - 9] and others). We will call these manifolds, as in [2], generalized Kenmotsu manifolds, and briefly GK-manifolds.

The following theorem holds.

Theorem 2.1 [19]. The complete group of structure equations for $G K$-manifolds on the space of the associated $G$-structure has the form:

$$
\text { 1) } d \omega=F_{a b} \omega^{a} \wedge \omega^{b}+F^{a b} \omega_{a} \wedge \omega_{b} \text {; }
$$

2) $d \omega^{a}=-\theta_{b}^{a} \wedge \omega^{b}+C^{a b c} \omega_{b} \wedge \omega_{c}-\frac{3}{2} F^{a b} \omega \wedge \omega_{b}+\delta_{b}^{a} \omega \wedge \omega^{b}$;

3) $d \omega_{a}=\theta_{a}^{b} \wedge \omega_{b}+C_{a b c} \omega^{b} \wedge \omega^{c}-\frac{3}{2} F_{a b} \omega \wedge \omega^{b}+\delta_{a}^{b} \omega \wedge \omega_{b}$;

4) $d \theta_{b}^{a}=-\theta_{c}^{a} \wedge \theta_{b}^{c}+\left(A_{b c}^{a d}-2 C^{a d h} C_{h b c}-\frac{3}{2} F^{a d} F_{b c}\right) \omega^{c} \wedge \omega_{d}+$

$$
\begin{gathered}
+\left(-\frac{1}{3} \delta_{b}^{a} F_{c d}+\frac{2}{3} \delta_{c}^{a} F_{d b}+\frac{2}{3} \delta_{d}^{a} F_{b c}\right) \omega^{c} \wedge \omega^{d}+ \\
+\left(\frac{1}{3} \delta_{b}^{a} F^{c d}-\frac{2}{3} \delta_{b}^{c} F^{d a}-\frac{2}{3} \delta_{b}^{d} F^{a c}\right) \omega_{c} \wedge \omega_{d}
\end{gathered}
$$

5) $d C^{a b c}+C^{d b c} \theta_{d}^{a}+C^{a d c} \theta_{d}^{b}+C^{a b d} \theta_{d}^{c}=C^{a b c d} \omega_{d}-2 \delta_{d}^{[a} F^{b c]} \omega^{d}-C^{a b c} \omega$;

6) $d C_{a b c}-C_{d b c} \theta_{a}^{d}-C_{a d c} \theta_{b}^{d}-C_{a b d} \theta_{c}^{d}=C_{a b c d} \omega^{d}-2 \delta_{[a}^{d} F_{b c]} \omega_{d}-C_{a b c} \omega$;

7) $d F^{a b}+F^{c b} \theta_{c}^{a}+F^{a c} \theta_{c}^{b}=-2 F^{a b} \omega$; 
8) $d F_{a b}-F_{c b} \theta_{a}^{c}-F_{a c} \theta_{b}^{c}=-2 F_{a b} \omega$.

where

$$
\begin{gathered}
A_{[b c]}^{a d}=A_{b c}^{[a d]}=0, C^{[a b c]}=C^{a b c}, C_{[a b c]}=C_{a b c}, \overline{C^{a b c}}=C_{a b c}, F^{a b}+F^{b a}= \\
0, F_{a b}+F_{b a}=0, \overline{F^{a b}}=F_{a b}, C^{a[b c d]}=\frac{3}{2} F^{a[b} F^{c d]}, C_{a[b c d]}= \\
\frac{3}{2} F_{a[b} F_{c d]}, F_{a d} C^{d b c}=0 .
\end{gathered}
$$

For generalized Kenmotsu varieties, the following fundamental identities hold [23].

Identity

$$
\mathrm{F}^{\mathrm{ad}} \mathrm{C}_{\mathrm{dbc}}=0
$$

will be called the first fundamental identity.

Identity

$$
\left(\mathrm{A}_{\mathrm{b}[\mathrm{c}}^{\mathrm{ag}}-2 \mathrm{C}^{\mathrm{agf}} \mathrm{C}_{\mathrm{fb}[\mathrm{c}}\right) \mathrm{C}_{|\mathrm{g}| \mathrm{dh}]}=0
$$

will be called the second fundamental identity.

Identity

$$
\left(\mathrm{A}_{\mathrm{b}[\mathrm{c}}^{\mathrm{ah}}-\frac{3}{2} \mathrm{~F}^{\mathrm{ah}} \mathrm{F}_{\mathrm{b}[\mathrm{c}}\right) \mathrm{F}_{|\mathrm{h}| \mathrm{d}]}=0
$$

will be called the third fundamental identity.

Identity

$$
2 \mathrm{~F}^{\mathrm{ab}} \mathrm{F}^{\mathrm{cd}}=\mathrm{F}^{\mathrm{ac}} \mathrm{F}^{\mathrm{db}}+\mathrm{F}^{\mathrm{ad}} \mathrm{F}^{\mathrm{bc}}
$$

will be called the fourth fundamental identity.

Identity

$$
\mathrm{C}_{\mathrm{abcg}} \mathrm{C}^{\mathrm{gdh}}=0
$$

will be called the fifth fundamental identity of GK-manifolds.

Relations (1.6) for GK-manifolds take the form:

$$
\begin{gathered}
\theta_{\hat{b}}^{a}=\frac{1}{2} F^{a b} \omega+C^{a b c} \omega_{c} ; \theta_{b}^{\hat{a}}=\frac{1}{2} F_{a b} \omega+C_{a b c} \omega^{c} ; \\
\theta_{0}^{a}=\delta_{b}^{a} \omega^{b}-F^{a b} \omega_{b} ; \theta_{0}^{\hat{a}}=\delta_{a}^{b} \omega_{b}-F_{a b} \omega^{b} ; \\
\theta_{a}^{0}=F_{a b} \omega^{b}-\delta_{a}^{b} \omega_{b} ; \theta_{\hat{a}}^{0}=F^{a b} \omega_{b}-\delta_{b}^{a} \omega^{b} .
\end{gathered}
$$

Consider the family of functions $C=\left\{C^{i}{ }_{j k}\right\} ; C^{a}{ }_{\hat{b} \hat{c}}=C^{a b c} ; C^{\hat{a}}{ }_{b c}=C_{a b c}$; all other components of the family $C$ are zero. From (2.2: 5-6) it follows that this system of functions, defined on the space of the associated $G$-structure, defines a globally defined tensor of type $(2,1)$ on the manifold $M$, which we call the first structure tensor of the GK-structure. Similarly, the family of functions $F=\left\{F_{j}^{i}\right\}, F^{a}{ }_{\hat{b}}=F^{a b}, F^{\hat{a}_{b}}=F_{a b}$, defined on the space of the associated $G$-structure, defines a globally defined tensor of type $(1,1)$ on the manifold $M$, which is called the second structure tensor of the $G K$-structure.

The first structure tensor of $G K$-manifolds has the following form:

$$
C(X, Y)=-\frac{1}{4}\left\{\Phi \circ \nabla_{\Phi Y}(\Phi) \Phi Y+\Phi^{2} \circ \nabla_{\Phi Y}(\Phi) \Phi^{2} X\right\} ; X, Y \in \mathcal{X}(M)
$$

The second structure tensor of $G K$-manifolds has the form:

$$
F(X)=-\nabla_{X} \xi ; X \in \mathcal{X}(M) .
$$


It is easy to check that the first and second structure tensors of $G K$-manifolds have the following properties:

$$
\begin{gathered}
\text { 1) } C(\xi, X)=C(X, \xi)=0 \text {; 2) } C(Y, X)=-C(X, Y) \\
\text { 3) } C(\Phi X, Y)=-C(X, \Phi Y)=-\Phi \circ C(X, Y) \text {; 4) } \eta \circ C(X, Y)=0 ; \\
\text { 5) }\langle\langle C(X, Y), Z\rangle\rangle+\overline{\langle\langle Y, C(X, Z)\rangle\rangle=0}
\end{gathered}
$$

6) $\Phi \circ F(X)=-F(\Phi X)$; 7) $F(\xi)=0$;8) $\eta \circ F=0$;

9) $\langle F(X), Y\rangle=-\langle X, F(Y)\rangle ; \quad X, Y, Z \in X(M)$.

Definition 2.2 [2, 12]. A GK-structure is called a special generalized Kenmotsu structure of the first kind (in short, an SGK-structure of the first kind) if $\mathrm{C}^{\mathrm{abc}}=\mathrm{C}_{\mathrm{abc}}=0$; a special generalized Kenmotsu structure of the second kind (in short, the SGK-structure of the second kind), if $F^{a b}=F_{a b}=0$. If $C^{a b c}=C_{a b c}=0$ and $F^{a b}=F_{a b}=0$, then the $G K$-structure is a Kenmotsu structure.

\section{Identities of curvature of GK-manifolds}

Let $M$ be a $G K$-variety. We recall the following theorem from ([13], [19]).

Theorem 3.1. The nonzero essential components of the Riemann-Christoffel tensor of a $G K$-manifold on the space of the associated $G$-structure have the form:

$$
\begin{gathered}
\text { 1) } R_{00 b}^{a}=F^{a c} F_{c b}+\delta_{b}^{a} ; R_{00 \hat{b}}^{\hat{a}}=F_{a c} F^{c b}+\delta_{a}^{b} ; \\
\text { 2) } R_{b c d}^{a}=-\frac{2}{3} \delta_{b}^{a} F_{c d}+\frac{1}{3} \delta_{c}^{a} F_{d b}+\frac{1}{3} \delta_{d}^{a} F_{b c} ; R_{\hat{b} \hat{c} \hat{d}}^{\hat{a}}=-\frac{2}{3} \delta_{a}^{b} F^{c d}+\frac{1}{3} \delta_{a}^{c} F^{d b}+ \\
\frac{1}{3} \delta_{a}^{d} F^{b c} ; \\
\text { 3) } R_{b c \hat{d}}^{a}=A_{b c}^{a d}-C^{a d h} C_{h b c}-\frac{1}{2} F^{a d} F_{b c}-\delta_{c}^{a} \delta_{b}^{d} ; R_{\hat{b} c \hat{d}}^{\hat{a}}=-A_{a c}^{b d}+C^{b d h} C_{h a c}+ \\
\frac{1}{2} F^{b d} F_{a c}+\delta_{c}^{b} \delta_{a}^{d} ; \\
\text { 4) } R_{\hat{b} c d}^{a}=2 C^{a b h} C_{h c d}+F^{a b} F_{c d}-2 \delta_{[c}^{a} \delta_{d]}^{b} ; R_{b \hat{c} \hat{d}}^{\hat{a}}=2 C_{a b h} C^{h c d}+F_{a b} F^{c d}- \\
2 \delta_{a}^{[c} \delta_{b}^{d]} ; \\
\text { 5) } R_{\hat{b} \hat{c} \hat{d}}^{a}=C^{a c d b}-\frac{1}{2}\left(F^{a b} F^{c d}+F^{a c} F^{d b}+F^{a d} F^{b c}\right) ; R_{b c d}^{\hat{a}}=C_{a c d b}- \\
\frac{1}{2}\left(F_{a b} F_{c d}+F_{a c} F_{d b}+F_{a d} F_{b c}\right) .
\end{gathered}
$$

The natural decomposition of the complexification of the module $\mathcal{X}(M)$ of an almost contact metric manifold $M$ into a direct sum $\chi(M)=D_{\Phi}^{\sqrt{-1}} \oplus D_{\Phi}^{-\sqrt{-1}} \oplus D_{\Phi}^{0}$ of the eigensubspaces of the structural endomorphism $\Phi$ corresponding to eigenvalues $\sqrt{-1}, \sqrt{-1}$ and 0 , respectively, determines the additional grading of the tensor algebra of this manifold. Each tensor of type $(r, 1)$ on $M$ considered as an $r$-linear mapping $T: \underbrace{\mathcal{X}(M) \times \mathcal{X}(M) \times \ldots \times \mathcal{X}(M)} \rightarrow \mathcal{X}(M)$ is naturally represented as a sum $3^{r}$ tensors of the same type, $C \otimes C^{\infty}(M)$-linear, or $C \otimes C^{\infty}(M)$-antilinear, or self-adjoint with respect to each argument. That is, if $T$ is a tensor of type $(r, 1)$ on $M$, then

$$
\begin{gathered}
T\left(X_{1}, \ldots, X_{r}\right)=(\sigma+\bar{\sigma}+m) \circ T\left(\sigma X_{1}+\bar{\sigma} X_{1}+m X_{1}, \ldots, \sigma X_{r}+\bar{\sigma} X_{r}+m X_{r}\right)= \\
\sigma \circ T\left(\sigma X_{1}, \sigma X_{2}, \ldots, \sigma X_{r}\right)+\sigma \circ T\left(\sigma X_{1}, \sigma X_{2}, \ldots, \sigma X_{r-1}, \bar{\sigma} X_{r}\right)+\sigma \circ
\end{gathered}
$$




$$
\begin{gathered}
T\left(\sigma X_{1}, \sigma X_{2}, \ldots, \sigma X_{r-1}, m X_{r}\right)+\cdots+m \circ T\left(m X_{1}, \ldots, \sigma X_{r}\right)+m \circ \\
T\left(m X_{1}, \ldots, \bar{\sigma} X_{r}\right)+m \circ T\left(m X_{1}, \ldots, m X_{r}\right) .
\end{gathered}
$$

The set of $3^{r+1}$ tensors of type $(r, 1)$ in the expansion (3.2) is called the spectrum of the tensor $T$, and these tensors themselves are called the elements of the spectrum. The element of the tensor spectrum is characterized by a decimal number, in which ternary units correspond to the numbers of linear arguments, two correspond to the numbers of antilinear arguments, and zeros correspond to the numbers of self-adjoint arguments. For example, $T$

characterizes the element of the spectrum of the tensor $T$, linear in the first and third arguments and antilinear in the second argument, since $16=1 \cdot 3^{2}+2 \cdot 3^{1}+1 \cdot 3^{0}$.

Consider the Riemannian curvature tensor $R$, a tensor of type $(3,1)$. On the space of the associated $G$-structure of an $A C$-manifold, the Riemannian curvature tensor is defined by 81 components $\left\{R_{j k l}^{i}\right\}$, where $i, j, k, l$ take the values $0, a, \hat{a}$. For example,

$$
\underbrace{R_{000}^{0}}_{(0)}=R_{000}^{0}, \underbrace{R_{00 a}^{0}}_{(1)}=R_{00 a}^{0}, \underbrace{R_{00 \hat{a}}^{0}}_{(2)}=R_{00 \hat{a}}^{0}, \ldots, \underbrace{R_{\hat{a} \hat{b} \hat{c}}^{\hat{d}}}_{(80)}=R_{\hat{a} \hat{b} \hat{c}}^{\hat{d}} .
$$

So for a $G K$-manifold, the Riemannian curvature tensor canonically be represented as the sum of the following nonzero elements of the spectrum:

$$
\begin{aligned}
& R_{1}=\underset{(11)}{R}=\left\{R_{a 0 \hat{b}}^{0}, R_{\hat{a} 0 b}^{0}\right\} ; R_{2}=\underset{(15)}{R}=\left\{R_{a \hat{b} 0}^{0}, R_{\hat{a} b 0}^{0}\right\} ; R_{3}=\underset{(28)}{R}=\left\{R_{00 b}^{a}, R_{00 \hat{b}}^{\hat{a}}\right\} ; R_{4}= \\
& \underset{(30)}{R}=\left\{R_{0 b 0}^{a}, R_{0 \hat{b} 0}^{\hat{a}}\right\} ; R_{5}=\underset{(40)}{R}=\left\{R_{b c d}^{a}, R_{\hat{b} \hat{c} \hat{d}}^{\hat{a}}\right\} ; R_{6}=\underset{(41)}{R}=\left\{R_{b c \hat{d}}^{a}, R_{\hat{b} \hat{c} c}^{\hat{a}}\right\} ; R_{7}=\underbrace{R}_{(43)}= \\
& \left\{R_{b \hat{c} d}^{a}, R_{\hat{b} c \hat{d}}^{\hat{a}}\right\} ; R_{8}=\underset{(49)}{R}=\left\{R_{\hat{b} c d}^{a}, R_{b \hat{c} \hat{d}}^{\hat{a}}\right\} ; R_{9}=\underset{(67)}{R}=\left\{R_{b c d}^{\hat{a}}, R_{\hat{b} \hat{c} \hat{d}}^{a}\right\} .
\end{aligned}
$$

The remaining components of the Riemannian curvature tensor are expressed in terms of those named because of its symmetry properties. Their vanishing sets, respectively, 9 invariant classes denoted by $\mathcal{R}_{1}, \mathcal{R}_{2}, \mathcal{R}_{3}, \mathcal{R}_{4}, \mathcal{R}_{5}, \mathcal{R}_{6}, \mathcal{R}_{7}, \mathcal{R}_{8}, \mathcal{R}_{9}$. Let us investigate in more detail the geometric meaning of the vanishing of invariants for a $G K$-manifold.

Definition 3.1. A $G K$-manifold for which $R_{i}=0$ is called $\boldsymbol{a}$ GK-manifold of the class $\mathcal{R}_{i}, i=1,2, \ldots, 9$.

Theorem 3.2. The invariant $R_{1}$ of the $G K$-manifold is calculated by the formula $R_{1}(X, Y)=R(\xi, \Phi X) \Phi Y ; \forall X, Y \in \mathcal{X}(M)$.

Proof. Taking into account (1.3), $\xi^{a}=\xi^{\hat{a}}=0, \xi^{0}=1$, and (3.1), we have $\{R(\xi, \Phi X) \Phi Y\}^{a}=R_{i j k}^{a} \xi^{j} \Phi_{l}^{k} X^{l} \Phi_{m}^{i} Y^{m}=R_{b c d}^{a} \xi^{c} \Phi_{l}^{d} X^{l} \Phi_{m}^{b} Y^{m}+R_{b c \hat{d}}^{a} \xi^{c} \Phi_{l}^{\widehat{d}} X^{l} \Phi_{m}^{b} Y^{m}+$ $R_{b c d}^{a} \xi^{\hat{c}} \Phi_{l}^{d} X^{l} \Phi_{m}^{b} Y^{m}+R_{\hat{b} c d}^{a} \xi^{c} \Phi_{l}^{d} X^{l} \Phi_{m}^{\hat{b}} Y^{m}+R_{0 b 0}^{a} \xi^{b} \Phi_{l}^{0} X^{l} \Phi_{m}^{0} Y^{0}+R_{00 b}^{a} \xi^{0} \Phi_{l}^{b} X^{l} \Phi_{m}^{0} Y^{m}=$ 0 . Similarly, $\{R(\xi, \Phi X) \Phi Y\}^{\hat{a}}=R_{i j k}^{\hat{a}} \xi^{j} \Phi_{l}^{k} X^{l} \Phi_{m}^{i} Y^{m}=0$. Further, $\{R(\xi, \Phi X) \Phi Y\}^{0}=$ $R_{i j k}^{0} \xi^{j} \Phi_{l}^{k} X^{l} \Phi_{m}^{i} Y^{m}=R_{b 0 \hat{c}}^{0} \xi^{0} \Phi_{l}^{\hat{c}} X^{l} \Phi_{m}^{b} Y^{m}+R_{b \hat{c} 0}^{0} \xi^{\hat{c}} \Phi_{l}^{0} X^{l} \Phi_{m}^{b} Y^{m}+R_{\hat{b} 0 c}^{0} \xi^{0} \Phi_{l}^{c} X^{l} \Phi_{m}^{\hat{b}} Y^{m}+$ $R_{\hat{b} c 0}^{0} \xi^{c} \Phi_{l}^{0} X^{l} \Phi_{m}^{\hat{b}} Y^{m}=R_{b 0 \hat{c}}^{0} X^{\hat{c}} Y^{b}+R_{\hat{b} 0 c}^{0} X^{c} Y^{\hat{b}}=R_{1}^{0}{ }_{b 0 \hat{c}}^{0} X^{\hat{c}} Y^{b}+R_{1 \hat{b} 0 c}^{0} X^{c} Y^{\hat{b}}=R_{1}(X, Y)$.

Let $M^{2 n+1}$ be a $G K$-manifold of the class $\mathcal{R}_{1}$. Then, according to Definition 3.1, $R_{1}=$ $\underbrace{R}_{w}=\left\{R_{a 0 \hat{b}}^{0}, R_{\hat{a} 0 b}^{0}\right\}=0$. Which, taking into account (3.1:1), gives $F^{a c} F_{c b}=-\delta_{b}^{a}$. Then the

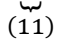

third fundamental identity will take the form:

$$
A_{b c}^{a h} F_{h d}-A_{b d}^{a h} F_{h c}+\frac{3}{2} \delta_{d}^{a} F_{b c}-\frac{3}{2} \delta_{c}^{a} F_{b d}=0 .
$$

We fold this identity over the indices a and $b$, then we get

$$
A_{a c}^{a h} F_{h d}-A_{a d}^{a h} F_{h c}-3 F_{c d}=0 .
$$


We contract this identity (3.3) in the indices a and c, then we obtain

$$
A_{b a}^{a h} F_{h d}-A_{b d}^{a h} F_{h a}+\frac{3}{2}(1-n) F_{b d}=0 .
$$

Thus, for a $G K$-manifold of class $\mathcal{R}_{1}$, the following equalities hold:

$$
\text { 1) } \left.F_{a b}=\frac{2}{3} A_{c[a}^{c d} F_{|d| b]} ; 2\right) F_{a b}=\frac{2}{3(n-1)}\left(A_{c a}^{c d} F_{d b}-A_{a b}^{c d} F_{d c}\right) \text {. }
$$

Theorem 3.3. The invariants $R_{2}, R_{3}, R_{4}$ of the $G K$-manifold are calculated by the formulas:

1) $R_{2}(X, Y)=R(\Phi X, \xi) \Phi Y$; 2) $R_{3}(X)=R(\xi, X) \xi$; 3) $R_{4}(X)=R(X, \xi) \xi ; \forall X, Y \in$ $x(M)$.

The proof is similar to the proof of Theorem 3.2.

Theorem 3.4. For $G K$-manifolds: 1) the class $\mathcal{R}_{1}$ coincides with the class $\mathcal{R}_{2} ; 2$ ) the $\mathcal{R}_{3}$ class is the same as the $\mathcal{R}_{4}$ class; 3 ) $\mathcal{R}_{6}$ class is the same as $\mathcal{R}_{7}$ class. A $G K$-manifold of class $\mathcal{R}_{6}$ is a $G K$-manifold of class $\mathcal{R}_{8}$.

The proof follows directly from the symmetry properties of the Riemannian curvature tensor, Definition 3.1 and Theorem 3.4.

Remark. Since, by virtue of the symmetry properties of the Riemannian curvature tensor, from $R_{a 0 \hat{b}}^{0}=0$ it follows that $R_{00 \hat{b}}^{\hat{a}}=0$, that is, a $G K$-variety of class $\mathcal{R}_{1}$ is a variety of class $\mathcal{R}_{3}$. The converse is also true.

According to Theorem 3.4, we call the invariants $\mathcal{R}_{1}, \mathcal{R}_{3}, \mathcal{R}_{6}, \mathcal{R}_{8}, \mathcal{R}_{9}$ the basic invariants of the GK-manifold. The following theorem gives explicit expressions for the main invariants of a $G K$-manifold.

Theorem 3.5.1) The invariant $R_{5}$ of the $G K$-manifold is calculated by the formula

$$
\begin{gathered}
R_{5}(X, Y) Z=\frac{1}{8}\left\{\Phi ^ { 2 } \circ \left[R\left(\Phi^{2} X, \Phi^{2} Y\right) \Phi^{2} Z-R\left(\Phi^{2} X, \Phi Y\right) \Phi Z-R\left(\Phi X, \Phi^{2} Y\right) \Phi Z-\right.\right. \\
\left.R(\Phi X, \Phi Y) \Phi^{2} Z\right]-\Phi \circ\left[R\left(\Phi^{2} X, \Phi^{2} Y\right) \Phi Z+R\left(\Phi^{2} X, \Phi Y\right) \Phi^{2} Z+R\left(\Phi X, \Phi^{2} Y\right) \Phi^{2} Z-\right. \\
R(\Phi X, \Phi Y) \Phi Z]\} ; \forall X, Y, Z \in \mathcal{X}(M) .
\end{gathered}
$$

2) The invariant $\mathcal{R}_{6}$ of the $G K$-manifold is calculated by the formula

$$
\begin{gathered}
R_{6}(X, Y) Z=\frac{1}{8}\left\{-\Phi^{2} \circ\left[R\left(\Phi^{2} X, \Phi^{2} Y\right) \Phi^{2} Z+R\left(\Phi^{2} X, \Phi Y\right) \Phi Z-R\left(\Phi X, \Phi^{2} Y\right) \Phi Z+\right.\right. \\
\left.R(\Phi X, \Phi Y) \Phi^{2} Z\right]+\Phi \circ\left[R\left(\Phi^{2} X, \Phi^{2} Y\right) \Phi Z-R\left(\Phi^{2} X, \Phi Y\right) \Phi^{2} Z+R\left(\Phi X, \Phi^{2} Y\right) \Phi^{2} Z+\right. \\
R(\Phi X, \Phi Y) \Phi Z]\} ; \forall X, Y, Z \in \mathcal{X}(M) .
\end{gathered}
$$

3) The invariant $\mathcal{R}_{8}$ of the $G K$-manifold is calculated by the formula

$$
\begin{gathered}
R_{8}(X, Y) Z=\frac{1}{8}\left\{-\Phi^{2} \circ\left[R\left(\Phi^{2} X, \Phi^{2} Y\right) \Phi^{2} Z+R\left(\Phi^{2} X, \Phi Y\right) \Phi Z+R\left(\Phi X, \Phi^{2} Y\right) \Phi Z-\right.\right. \\
\left.R(\Phi X, \Phi Y) \Phi^{2} Z\right]-\Phi \circ\left[R\left(\Phi^{2} X, \Phi^{2} Y\right) \Phi Z-R\left(\Phi^{2} X, \Phi Y\right) \Phi^{2} Z-R\left(\Phi X, \Phi^{2} Y\right) \Phi^{2} Z-\right. \\
R(\Phi X, \Phi Y) \Phi Z]\} ; \forall X, Y, Z \in \mathcal{X}(M) .
\end{gathered}
$$

4) The invariant $\mathcal{R}_{9}$ of the $G K$-manifold is calculated by the formula

$$
\begin{gathered}
R_{9}(X, Y) Z=\frac{1}{8}\left\{\Phi ^ { 2 } \circ \left[-R\left(\Phi^{2} X, \Phi^{2} Y\right) \Phi^{2} Z+R\left(\Phi^{2} X, \Phi Y\right) \Phi Z+R\left(\Phi X, \Phi^{2} Y\right) \Phi Z+\right.\right. \\
\left.R(\Phi X, \Phi Y) \Phi^{2} Z\right]-\Phi \circ\left[R\left(\Phi^{2} X, \Phi^{2} Y\right) \Phi Z+R\left(\Phi^{2} X, \Phi Y\right) \Phi^{2} Z+R\left(\Phi X, \Phi^{2} Y\right) \Phi^{2} Z-\right. \\
R(\Phi X, \Phi Y) \Phi Z]\} ; \forall X, Y, Z \in \mathcal{X}(M) .
\end{gathered}
$$

Proof. We carry out the proof for case 1). The rest of the cases are proved similarly. Taking into account (1.3) and (3.1), we have: 


$$
\begin{aligned}
& \left(R\left(\Phi^{2} X, \Phi^{2} Y\right) \Phi^{2} Z\right)^{a}=R_{i j k}^{a}\left(\Phi^{2} X\right)^{j}\left(\Phi^{2} Y\right)^{k}\left(\Phi^{2} Z\right)^{i}=-R_{b c d}^{a} X^{c} Y^{d} Z^{b}-R_{b c \hat{d}}^{a} X^{c} Y^{\hat{d}} Z^{b}- \\
& R_{b c d}^{a} X^{\hat{c}} Y^{d} Z^{b}-R_{b c \hat{c} \hat{d}}^{a} X^{\hat{c}} Y^{\hat{d}} Z^{b}-R_{\hat{b} c d}^{a} X^{c} Y^{d} Z^{\hat{b}}-R_{\hat{b} c \hat{d}}^{a} X^{c} Y^{\hat{d}} Z^{\hat{b}}-R_{\hat{b} c d}^{a} X^{\hat{c}} Y^{d} Z^{\hat{b}}- \\
& R_{\hat{b} \hat{c} \hat{d}}^{a} X^{\hat{c}} Y^{\hat{d}} Z^{\hat{b}} \\
& \left(R\left(\Phi^{2} X, \Phi Y\right) \Phi Z\right)^{a}=R_{i j k}^{a}\left(\Phi^{2} X\right)^{j}(\Phi Y)^{k}(\Phi Z)^{i}=R_{b c d}^{a} X^{c} Y^{d} Z^{b}-R_{b c \hat{d}}^{a} X^{c} Y^{\hat{d}} Z^{b}+ \\
& R_{b c d}^{a} X^{\hat{c}} Y^{d} Z^{b}-R_{b c \hat{d}}^{a} X^{\hat{c}} Y^{\hat{d}} Z^{b}-R_{\hat{b} c d}^{a} X^{c} Y^{d} Z^{\hat{b}}+R_{\hat{b} c \hat{d}}^{a} X^{c} Y^{\hat{d}} Z^{\hat{b}}-R_{\hat{b} c d}^{a} X^{\hat{C}} Y^{d} Z^{\hat{b}}+ \\
& R_{\hat{b} \hat{c} \hat{d}}^{a} X^{\hat{c}} Y^{\hat{d}} Z^{\hat{b}} \\
& \left(R\left(\Phi X, \Phi^{2} Y\right) \Phi Z\right)^{a}=R_{i j k}^{a}(\Phi X)^{j}\left(\Phi^{2} Y\right)^{k}(\Phi Z)^{i}=R_{b c d}^{a} X^{c} Y^{d} Z^{b}+R_{b c \hat{d}}^{a} X^{c} Y^{\hat{d}} Z^{b}- \\
& R_{b \hat{b} d}^{a} X^{\hat{c}} Y^{d} Z^{b}-R_{b \hat{c} \hat{d}}^{a} X^{\hat{c}} Y^{\hat{d}} Z^{b}-R_{\hat{b} c d}^{a} X^{c} Y^{d} Z^{\hat{b}}-R_{\hat{b} c \hat{d}}^{a} X^{c} Y^{\hat{d}} Z^{\hat{b}}+R_{\hat{b} \hat{c} d}^{a} X^{\hat{c}} Y^{d} Z^{\hat{b}}+ \\
& R_{\hat{b} \hat{d}}^{a} \hat{d} X^{\hat{C}} Y^{\hat{d}} Z^{\hat{b}} \\
& \left(R(\Phi X, \Phi Y) \Phi^{2} Z\right)^{a}=R_{i j k}^{a}(\Phi X)^{j}(\Phi Y)^{k}\left(\Phi^{2} Z\right)^{i}=R_{b c d}^{a} X^{c} Y^{d} Z^{b}-R_{b c \hat{d}}^{a} X^{c} Y^{\hat{d}} Z^{b}- \\
& R_{b c d}^{a} X^{\hat{c}} Y^{d} Z^{b}+R_{b c \hat{d}}^{a} X^{\hat{c}} Y^{\hat{d}} Z^{b}+R_{\hat{b} c d}^{a} X^{c} Y^{d} Z^{\hat{b}}-R_{\hat{b} c \hat{d}}^{a} X^{c} Y^{\hat{d}} Z^{\hat{b}}-R_{\hat{b} c d}^{a} X^{\hat{c}} Y^{d} Z^{\hat{b}}+ \\
& R_{\hat{b} \hat{d} \hat{d}}^{a} X^{\hat{c}} Y^{\hat{a}} Z^{\hat{b}} .
\end{aligned}
$$

Taking these relations into account, we obtain

$$
\begin{aligned}
& \left\{R\left(\Phi^{2} X, \Phi^{2} Y\right) \Phi^{2} Z-R\left(\Phi^{2} X, \Phi Y\right) \Phi Z-R\left(\Phi X, \Phi^{2} Y\right) \Phi Z-R(\Phi X, \Phi Y) \Phi^{2} Z\right\}^{a} \\
& =-R_{b c d}^{a} X^{c} Y^{d} Z^{b}-R_{b c \hat{d}}^{a} X^{c} Y^{\hat{d}} Z^{b}- \\
& -R_{b c \hat{c} d}^{a} X^{\hat{c}} Y^{d} Z^{b}-R_{b \hat{c} \hat{d}}^{a} X^{\hat{c}} Y^{\hat{d}} Z^{b}-R_{\hat{b} c d}^{a} X^{c} Y^{d} Z^{\hat{b}}-R_{\hat{b} c \hat{d} \hat{d}}^{a} X^{c} Y^{\hat{d}} Z^{\hat{b}}-R_{\hat{b} c d}^{a} X^{\hat{c}} Y^{d} Z^{\hat{b}}- \\
& -R_{\hat{b} c \hat{d} \hat{d}}^{a} X^{\hat{c}} Y^{\hat{d}} Z^{\hat{b}}-R_{b c d}^{a} X^{c} Y^{d} Z^{b}+R_{b c \hat{d}}^{a} X^{c} Y^{\hat{d}} Z^{b}-R_{b c \hat{c} d}^{a} X^{\hat{c}} Y^{d} Z^{b}+R_{b c \hat{d} \hat{d}}^{a} X^{\hat{c}} Y^{\hat{d}} Z^{b}+ \\
& +R_{\hat{b} c d}^{a} X^{c} Y^{d} Z^{\hat{b}}-R_{\hat{b} c \hat{d}}^{a} X^{c} Y^{d} Z^{\hat{b}}+R_{\hat{b} c d}^{a} X^{\hat{C}} Y^{d} Z^{\hat{b}}-R_{\hat{b} c \hat{a}}^{a} X^{\hat{c}} Y^{\hat{d}} Z^{\hat{b}}-R_{b c d}^{a} X^{c} Y^{d} Z^{b}- \\
& -R_{b c \hat{d}}^{a} X^{c} Y^{\hat{d}} Z^{b}+R_{b c \hat{c} d}^{a} X^{\hat{c}} Y^{d} Z^{b}+R_{b \hat{c} \hat{d}}^{a} X^{\hat{c}} Y^{\hat{d}} Z^{b}+R_{\hat{b} c d}^{a} X^{c} Y^{d} Z^{\hat{b}}+R_{\hat{b} c \hat{d}}^{a} X^{c} Y^{\hat{d}} Z^{\hat{b}}+ \\
& R_{\hat{b} \hat{c} d}^{a} X^{\hat{c}} Y^{d} Z^{\hat{b}}-R_{\hat{b} \hat{d}}^{a} X^{\hat{c}} Y^{\hat{d}} Z^{\hat{b}}-R_{b c d}^{a} X^{c} Y^{d} Z^{b}+R_{b c \hat{d}}^{a} X^{c} Y^{\hat{d}} Z^{b}+R_{b c d}^{a} X^{\hat{c}} Y^{d} Z^{b}- \\
& R_{b c \hat{d}}^{a} X^{\hat{c}} Y^{\hat{d}} Z^{b}-R_{\hat{b} c d}^{a} X^{c} Y^{d} Z^{\hat{b}}+R_{\hat{b} c \hat{d}}^{a} X^{c} Y^{\hat{d}} Z^{\hat{b}}+R_{\hat{b} c d}^{a} X^{\hat{c}} Y^{d} Z^{\hat{b}}-R_{\hat{b} \hat{c} \hat{d}}^{a} X^{\hat{c}} Y^{\hat{d}} Z^{\hat{b}}= \\
& -4 R_{b c d}^{a} X^{c} Y^{d} Z^{b}-4 R_{\hat{b} \hat{c} \hat{d}}^{a} X^{\hat{c}} Y^{\hat{d}} Z^{\hat{b}} \text {. }
\end{aligned}
$$

For expression

$$
\begin{gathered}
R\left(\Phi^{2} X, \Phi^{2} Y\right) \Phi Z+R\left(\Phi^{2} X, \Phi Y\right) \Phi^{2} Z+R\left(\Phi X, \Phi^{2} Y\right) \Phi^{2} Z-R(\Phi X, \Phi Y) \Phi Z ; \forall X, Y, Z \\
\in \mathcal{X}(M)
\end{gathered}
$$

we have:

$$
\begin{aligned}
& \left(R\left(\Phi^{2} X, \Phi^{2} Y\right) \Phi Z\right)^{a}=R_{i j k}^{a}\left(\Phi^{2} X\right)^{j}\left(\Phi^{2} Y\right)^{k}(\Phi Z)^{i}=\sqrt{-1} R_{b c d}^{a} X^{c} Y^{d} Z^{b}+ \\
& \sqrt{-1} R_{b c \hat{d}}^{a} X^{c} Y^{\hat{d}} Z^{b}+\sqrt{-1} R_{b c d}^{a} X^{\hat{c}} Y^{d} Z^{b}+\sqrt{-1} R_{b \hat{c} \hat{d}}^{a} X^{\hat{c}} Y^{\hat{a}} Z^{b}-\sqrt{-1} R_{\hat{b} c d}^{a} X^{c} Y^{d} Z^{\hat{b}}- \\
& \sqrt{-1} R_{\hat{b} c \hat{d}}^{a} X^{c} Y^{\hat{d}} Z^{\hat{b}}-\sqrt{-1} R_{\hat{b} \hat{c} d}^{a} X^{\hat{C}} Y^{d} Z^{\hat{b}}-\sqrt{-1} R_{\hat{b} \hat{c} \hat{d}}^{a} X^{\hat{c}} Y^{\hat{d}} Z^{\hat{b}} ; \\
& \left(R\left(\Phi^{2} X, \Phi Y\right) \Phi^{2} Z\right)^{a}=R_{i j k}^{a}\left(\Phi^{2} X\right)^{j}(\Phi Y)^{k}\left(\Phi^{2} Z\right)^{i}=\sqrt{-1} R_{b c d}^{a} X^{c} Y^{d} Z^{b}- \\
& \sqrt{-1} R_{b c \hat{d}}^{a} X^{c} Y^{\hat{d}} Z^{b}+\sqrt{-1} R_{b \hat{c} d}^{a} X^{\hat{c}} Y^{d} Z^{b}-\sqrt{-1} R_{b \hat{c} \hat{d}}^{a} X^{\hat{c}} Y^{\hat{d}} Z^{b}+\sqrt{-1} R_{\hat{b} c d}^{a} X^{c} Y^{d} Z^{\hat{b}}- \\
& \sqrt{-1} R_{\hat{b} c \hat{d}}^{a} X^{c} Y^{\hat{d}} Z^{\hat{b}}+\sqrt{-1} R_{\hat{b} \hat{c} d}^{a} X^{\hat{c}} Y^{d} Z^{\hat{b}}-\sqrt{-1} R_{\hat{b} \hat{c} \hat{d}}^{a} X^{\hat{c}} Y^{\hat{d}} Z^{\hat{b}} ; \\
& \left(R\left(\Phi X, \Phi^{2} Y\right) \Phi^{2} Z\right)^{a}=R_{i j k}^{a}(\Phi X)^{j}\left(\Phi^{2} Y\right)^{k}\left(\Phi^{2} Z\right)^{i}=\sqrt{-1} R_{b c d}^{a} X^{c} Y^{d} Z^{b}+ \\
& \sqrt{-1} R_{b c \hat{d}}^{a} X^{c} Y^{\hat{d}} Z^{b}-\sqrt{-1} R_{b \hat{c} d}^{a} X^{\hat{c}} Y^{d} Z^{b}-\sqrt{-1} R_{b \hat{c} \hat{d}}^{a} X^{\hat{c}} Y^{\hat{d}} Z^{b}+\sqrt{-1} R_{\hat{b} c d}^{a} X^{c} Y^{d} Z^{\hat{b}}+ \\
& \sqrt{-1} R_{\hat{b} c \hat{d}}^{a} X^{c} Y^{\hat{d}} Z^{\hat{b}}-\sqrt{-1} R_{\hat{b} \hat{c} d}^{a} X^{\hat{c}} Y^{d} Z^{\hat{b}}-\sqrt{-1} R_{\hat{b} \hat{c} \hat{d}}^{a} X^{\hat{c}} Y^{\hat{d}} Z^{\hat{b}}
\end{aligned}
$$




$$
\begin{gathered}
(R(\Phi X, \Phi Y) \Phi Z)^{a}=R_{i j k}^{a}(\Phi X)^{j}(\Phi Y)^{k}(\Phi Z)^{i}=-\sqrt{-1} R_{b c d}^{a} X^{c} Y^{d} Z^{b}+ \\
\sqrt{-1} R_{b c \hat{d}}^{a} X^{c} Y^{\hat{a}} Z^{b}+\sqrt{-1} R_{b c d}^{a} X^{\hat{c}} Y^{d} Z^{b}-\sqrt{-1} R_{b \hat{a}}^{a} X^{\hat{C}} Y^{\hat{a}} Z^{b}+\sqrt{-1} R_{\hat{b} c d}^{a} X^{c} Y^{d} Z^{\hat{b}}- \\
\sqrt{-1} R_{\hat{b} c \hat{d}}^{a} X^{c} Y^{\hat{d}} Z^{\hat{b}}-\sqrt{-1} R_{\hat{b} \hat{c} d}^{a} X^{\hat{c}} Y^{d} Z^{\hat{b}}+\sqrt{-1} R_{\hat{b} \hat{c} \hat{d}}^{a} X^{\hat{c}} Y^{\hat{d}} Z^{\hat{b}} .
\end{gathered}
$$

Thus,

$$
\begin{aligned}
& \left\{R\left(\Phi^{2} X, \Phi^{2} Y\right) \Phi Z+R\left(\Phi^{2} X, \Phi Y\right) \Phi^{2} Z+R\left(\Phi X, \Phi^{2} Y\right) \Phi^{2} Z-R(\Phi X, \Phi Y) \Phi Z\right\}^{a} \\
& =\sqrt{-1} R_{b c d}^{a} X^{c} Y^{d} Z^{b}+\sqrt{-1} R_{b c \hat{d}}^{a} X^{c} Y^{\hat{d}} Z^{b}+ \\
& +\sqrt{-1} R_{b \hat{c} d}^{a} X^{\hat{c}} Y^{d} Z^{b}+\sqrt{-1} R_{b \hat{c} \hat{d}}^{a} X^{\hat{c}} Y^{\hat{a}} Z^{b}-\sqrt{-1} R_{\hat{b} c d}^{a} X^{c} Y^{d} Z^{\hat{b}}-\sqrt{-1} R_{\hat{b} c \hat{d}}^{a} X^{c} Y^{\hat{a}} Z^{\hat{b}} \\
& -\sqrt{-1} R_{\hat{b} \hat{c} d}^{a} X^{\hat{c}} Y^{d} Z^{\hat{b}}-\sqrt{-1} R_{\hat{b} \hat{c} \hat{d}}^{a} X^{\hat{c}} Y^{\hat{d}} Z^{\hat{b}}+ \\
& +\sqrt{-1} R_{b c d}^{a} X^{c} Y^{d} Z^{b}-\sqrt{-1} R_{b c \hat{d}}^{a} X^{c} Y^{\hat{d}} Z^{b}+\sqrt{-1} R_{b c d}^{a} X^{\hat{c}} Y^{d} Z^{b}-\sqrt{-1} R_{b \hat{c} \hat{d}}^{a} X^{\hat{c}} Y^{\hat{d}} Z^{b} \\
& +\sqrt{-1} R_{\hat{b} c d}^{a} X^{c} Y^{d} Z^{\hat{b}}-\sqrt{-1} R_{\hat{b} c \hat{d}}^{a} X^{c} Y^{\hat{d}} Z^{\hat{b}}+\sqrt{-1} R_{\hat{b} c d}^{a} X^{\hat{c}} Y^{d} Z^{\hat{b}} \\
& -\sqrt{-1} R_{\hat{b} \hat{c} \hat{d}}^{a} X^{\hat{c}} Y^{\hat{d}} Z^{\hat{b}}+\sqrt{-1} R_{b c d}^{a} X^{c} Y^{d} Z^{b}+ \\
& +\sqrt{-1} R_{b c \hat{d}}^{a} X^{c} Y^{\hat{d}} Z^{b}-\sqrt{-1} R_{b c d}^{a} X^{\hat{c}} Y^{d} Z^{b}-\sqrt{-1} R_{b \hat{c} \hat{d}}^{a} X^{\hat{c}} Y^{\hat{d}} Z^{b}+\sqrt{-1} R_{\hat{b} c d}^{a} X^{c} Y^{d} Z^{\hat{b}} \\
& +\sqrt{-1} R_{\hat{b} c \hat{d}}^{a} X^{c} Y^{\hat{d}} Z^{\hat{b}}-\sqrt{-1} R_{\hat{b} c d}^{a} X^{\hat{c}} Y^{d} Z^{\hat{b}}-\sqrt{-1} R_{\hat{b} \hat{c} \hat{d}}^{a} X^{\hat{c}} Y^{\hat{d}} Z^{\hat{b}} \\
& +\sqrt{-1} R_{b c d}^{a} X^{c} Y^{d} Z^{b}-\sqrt{-1} R_{b c \hat{d}}^{a} X^{c} Y^{\hat{d}} Z^{b}- \\
& -\sqrt{-1} R_{b c d}^{a} X^{\hat{c}} Y^{d} Z^{b}+\sqrt{-1} R_{b c \hat{a}}^{a} X^{\hat{c}} Y^{\hat{d}} Z^{b}-\sqrt{-1} R_{\hat{b} c d}^{a} X^{c} Y^{d} Z^{\hat{b}}+\sqrt{-1} R_{\hat{b} c \hat{d}}^{a} X^{c} Y^{\hat{d}} Z^{\hat{b}}+ \\
& \sqrt{-1} R_{\hat{b} c d}^{a} X^{\hat{c}} Y^{d} Z^{\hat{b}}-\sqrt{-1} R_{\hat{b} \hat{d}}^{a} X^{\hat{C}} Y^{\hat{d}} Z^{\hat{b}}=4 \sqrt{-1} R_{b c d}^{a} X^{c} Y^{d} Z^{b}-4 \sqrt{-1} R_{\hat{b} \hat{c} \hat{d}}^{a} X^{\hat{c}} Y^{\hat{d}} Z^{\hat{b}} .
\end{aligned}
$$

So,

$\frac{1}{8}\left\{\Phi^{2} \circ\left[R\left(\Phi^{2} X, \Phi^{2} Y\right) \Phi^{2} Z-R\left(\Phi^{2} X, \Phi Y\right) \Phi Z-R\left(\Phi X, \Phi^{2} Y\right) \Phi Z-R(\Phi X, \Phi Y) \Phi^{2} Z\right]-\right.$ $\left.\Phi \circ\left[R\left(\Phi^{2} X, \Phi^{2} Y\right) \Phi Z+R\left(\Phi^{2} X, \Phi Y\right) \Phi^{2} Z+R\left(\Phi X, \Phi^{2} Y\right) \Phi^{2} Z-R(\Phi X, \Phi Y) \Phi Z\right]\right\}^{a}=$ $\frac{1}{8}\left\{\Phi^{2} \circ\left[R\left(\Phi^{2} X, \Phi^{2} Y\right) \Phi^{2} Z-R\left(\Phi^{2} X, \Phi Y\right) \Phi Z-R\left(\Phi X, \Phi^{2} Y\right) \Phi Z-R(\Phi X, \Phi Y) \Phi^{2} Z\right]\right\}^{a}-$ $\frac{1}{8}\left\{\Phi \circ\left[R\left(\Phi^{2} X, \Phi^{2} Y\right) \Phi Z+R\left(\Phi^{2} X, \Phi Y\right) \Phi^{2} Z+R\left(\Phi X, \Phi^{2} Y\right) \Phi^{2} Z-R(\Phi X, \Phi Y) \Phi Z\right]\right\}^{a}=$

$$
\begin{gathered}
\frac{1}{8}\left(4 R_{b c d}^{a} X^{c} Y^{d} Z^{b}+4 R_{\hat{b} c \hat{a}}^{a} X^{\hat{c}} Y^{\hat{d}} Z^{\hat{b}}\right)-\frac{1}{8}\left(-4 R_{b c d}^{a} X^{c} Y^{d} Z^{b}+4 R_{\hat{b} \hat{c} \hat{d}}^{a} X^{\hat{c}} Y^{\hat{d}} Z^{\hat{b}}\right)= \\
R_{b c d}^{a} X^{c} Y^{d} Z^{b}=\left\{R_{5}(X, Y) Z\right\}^{a} .
\end{gathered}
$$

Similarly, we obtain

$\frac{1}{8}\left\{\Phi^{2} \circ\left[R\left(\Phi^{2} X, \Phi^{2} Y\right) \Phi^{2} Z-R\left(\Phi^{2} X, \Phi Y\right) \Phi Z-R\left(\Phi X, \Phi^{2} Y\right) \Phi Z-R(\Phi X, \Phi Y) \Phi^{2} Z\right]-\right.$ $\left.\Phi \circ\left[R\left(\Phi^{2} X, \Phi^{2} Y\right) \Phi Z+R\left(\Phi^{2} X, \Phi Y\right) \Phi^{2} Z+R\left(\Phi X, \Phi^{2} Y\right) \Phi^{2} Z-R(\Phi X, \Phi Y) \Phi Z\right]\right\}^{\hat{a}}=$

$$
R_{\hat{b} \hat{c} \hat{d}}^{\hat{a}} X^{c} Y^{d} Z^{b}=\left\{R_{5}(X, Y) Z\right\}^{\hat{a}} .
$$

Thus, the functions $\left\{R_{b c d}^{a}\right\}$ and $\left\{R_{\hat{b} c \hat{c}}^{\hat{a}}\right\}$ are components of the tensor

$$
\begin{gathered}
\frac{1}{8}\left\{\Phi^{2} \circ\left[R\left(\Phi^{2} X, \Phi^{2} Y\right) \Phi^{2} Z-R\left(\Phi^{2} X, \Phi Y\right) \Phi Z-R\left(\Phi X, \Phi^{2} Y\right) \Phi Z-R(\Phi X, \Phi Y) \Phi^{2} Z\right]-\right. \\
\left.\Phi \circ\left[R\left(\Phi^{2} X, \Phi^{2} Y\right) \Phi Z+R\left(\Phi^{2} X, \Phi Y\right) \Phi^{2} Z+R\left(\Phi X, \Phi^{2} Y\right) \Phi^{2} Z-R(\Phi X, \Phi Y) \Phi Z\right]\right\},
\end{gathered}
$$

and therefore this tensor coincides with the tensor $R_{5}(X, Y) Z$, as required

Theorem 3.6. A $G K$-manifold of class $R_{5}$ is a Kenmotsu variety, that is, a manifold obtained from a cosymplectic manifold by a canonical concircular transformation of a cosymplectic structure.

Proof. Let $M$ be a $G K$-manifold of class $R_{5}$, that is, $R_{5}=\underset{(40)}{R}=\left\{R_{b c d}^{a}, R_{\hat{b} \hat{c} \hat{c}}^{\hat{a}}\right\}=0$. By virtue of (3.1:2), this is equivalent to the fact that $-\frac{2}{3} \delta_{b}^{a} F_{c d}+\frac{1}{3} \delta_{c}^{a} F_{d b}+\frac{1}{3} \delta_{d}^{a} F_{b c}=0$. Let us 
collapse this equality by indexes $a$ and $b$, then $2 n F_{c d}=F_{d c}+F_{d c} \Leftrightarrow 2 n F_{c d}=2 F_{d c} \Leftrightarrow$ $2(n+1) F_{c d}=0 \Leftrightarrow F_{c d}=0$.

Thus, a $G K$-manifold of class $R_{5}$ is a Kenmotsu manifold. Therefore, according to [24], it is a manifold obtained from a cosymplectic manifold by a canonical concircular transformation of a cosymplectic structure.

Theorem 3.7. A $G K$-manifold of class $R_{6}$ is a special generalized Kenmotsu manifold of the second type.

Proof. Let $M$ be a GK-manifold of class $R_{6}$, that is, $R_{6}=\underset{(41)}{R}=\left\{R_{b c \hat{d}}^{a}, R_{\hat{b} \hat{c} d}^{\hat{a}}\right\}=0$. By virtue of (3.1:3) this is equivalent to $A_{b c}^{a d}=C^{a d h} C_{h b c}+\frac{1}{2} F^{a d} F_{b c}+\delta_{c}^{a} \delta_{b}^{d}$. Symmetrying this identity first over the indices $a$ and $d$, and then over the indices $b$ and $c$, we obtain, taking into account (2.3), $A_{b c}^{a d}=\frac{1}{2} \tilde{\delta}_{b c}^{a d}$, where $\tilde{\delta}_{b c}^{a d}=\delta_{b}^{a} \delta_{c}^{d}+\delta_{c}^{a} \delta_{b}^{d}$. Then the third fundamental identity can be written in the form $\left(\frac{1}{2} \tilde{\delta}_{b[c}^{a h}-\frac{3}{2} F^{a h} F_{b[c}\right) F_{|h| d]}=0$, that is, $2 \delta_{b}^{a} F_{c d}-\delta_{c}^{a} F_{d b}-$ $\delta_{d}^{a} F_{b c}=3 F^{a h}\left(F_{b c} F_{h d}-F_{b d} F_{h c}\right)$. Let us collapse the obtained equality by indices $a$ and $b$, then we get $2(n+1) F_{c d}=3 F^{a h}\left(F_{a c} F_{h d}-F_{a d} F_{h c}\right)$, i.e., taking into account (2.3), $F_{c d}=$ $\frac{3}{2(n+1)} F^{a h}\left(F_{a c} F_{h d}-F_{a d} F_{h c}\right)$. Due to the fourth fundamental identity, the last identity takes the form: $F_{c d}=\frac{3}{2(n+1)} F^{a h} F_{a h} F_{c d}$, i.e. $F_{c d}\left(1+\frac{3}{2(n+1)} F^{a h} F_{a h}\right)=0$. From the obtained equality, we have that $F_{c d}=0$. And by Definition 2.2, a $G K$-manifold of class $R_{6}$ is a special generalized Kenmotsu variety of the second type.

Using the results on $S G K$-manifolds of the second type obtained in [2], [19], Theorem 3.7 can be formulated as:

Theorem 3.8. A $G K$-manifold of class $R_{6}$ coincides with an almost contact metric manifold obtained from the most exact cosymplectic manifold by the canonical concircular transformation of the most exact cosymplectic structure.

Theorem 3.9. A $G K$-manifold of class $R_{8}$ is a special generalized Kenmotsu manifold of the second type.

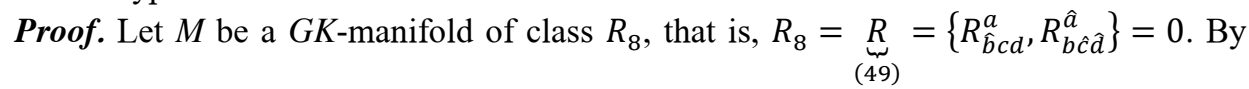
virtue of (3.1:3), this is equivalent to the fact that $2 C^{a b h} C_{h c d}+F^{a b} F_{c d}-2 \delta_{[c}^{a} \delta_{d]}^{b}=0$, that is,

$$
2 C^{a b h} C_{h c d}+F^{a b} F_{c d}=2 \delta_{[c}^{a} \delta_{d]}^{b} .
$$

Let us alternate identity (3.7) by indices $a$ and $b$, then, taking into account (2.3), we obtain: $2 C^{a b h} C_{h c d}+F^{a b} F_{c d}=2 \delta_{[c}^{[a} \delta_{d]}^{b]}$. We contract the resulting identity with the object $F^{d f}$, then, taking into account the first fundamental identity, we obtain $F^{a b} F_{c d} F^{d f}=\delta_{c}^{a} F^{b f}-\delta_{c}^{b} F^{a f}$. We contract the resulting equality by indices $c$ and $f$, then we get

$$
F^{a b} \sum_{c d}\left|F_{c d}\right|^{2}=2 F^{a b} .
$$

The last equality implies:

1) $F^{a b}=0$, i.e. the manifold $M$ is an $S G K$-manifold of the second type;

2) $\sum_{c d}\left|F_{c d}\right|^{2}=2$.

In the second case, consider the third fundamental identity, i.e. $A_{b[c}^{a h} F_{|h| d]}=$ $\frac{3}{2} F^{a h} F_{b[c} F_{|h| d]}$. Let us collapse this equality by indices $a$ and $d$, then $A_{b c}^{a h} F_{h a}-A_{b a}^{a h} F_{h c}=$ $\frac{3}{2} F^{a h}\left(F_{b c} F_{h a}-F_{b a} F_{h c}\right)$. An alternation of the latter identity with respect to indices $\mathrm{b}$ and $\mathrm{c}$, by virtue of (2.3), gives 


$$
A_{a[b}^{a h} F_{|h| c]}=-\frac{3}{2} F^{a h} F_{a[b} F_{|h| c]} .
$$

We collapse the third fundamental identity by indices $a$ and $b$, then we get $A_{a c}^{a h} F_{h d}-$ $A_{a d}^{a h} F_{h c}=\frac{3}{2} F^{a h}\left(F_{a c} F_{h d}-F_{a d} F_{h c}\right)$. Renaming the indices in the resulting equality, we obtain

$$
A_{a[b}^{a d} F_{|d| c]}=\frac{3}{2} F^{a d} F_{a[b} F_{|d| c]} .
$$

It follows from (3.9) and (3.10) that $F^{a d} F_{a[b} F_{|d| c]}=0$. The resulting equality, due to the fourth fundamental identity, can be written in the form $F^{a d} F_{a d} F_{b c}=0$, which, due to the condition $\sum_{c d}\left|F_{c d}\right|^{2}=2$, will be written in the form $2 F_{b c}=0$. Thus, $F_{b c}=0$, i.e. the manifold is a special generalized Kenmotsu manifold of the second type.

Taking into account that an $S G K$-structure of the second type is obtained by the canonical concircular transformation of the most exact cosymplectic structure, and using the local structure of the most exact cosymplectic manifold [25], Theorem 3.9 can be formulated as follows:

Theorem 3.10. A simply connected $G K$-manifold of class $R_{8}$ is canonically concircular to the product of an approximately Kähler manifold and the real line.

\section{References}

1. A. Shukla, Kuwait J. Sci. Eng. 23(2), 139-144 (1996)

2. S.V. Umnova, Geometry of Kenmotsu manifolds and their generalizations: Dis.... Cand. physical-mat. sciences (Moscow State Pedagogical University, Moscow, 2002)

3. M.M. Tripathi, S.S. Shukla Bull. Cal. Math. Soc. 95(1), 17-30 (2003)

4. V.A. Khan, M.A. Khan, S. Uddin, Soochow Journal of Mathematics 33(4), $563-568$ (2010)

5. A. Mobin, Jae-Bok Jun, Journal of the Chungcheong Mathematical Society 23(2), 257 266 (2010)

6. A. Mobin, Mathematicians Vesnik 62(3), 189 - 198 (2010)

7. M. Ahmad, Jae-Bok Jun, J. Korean Soc. Math. Educ. Ser. B: Pure Appl. Math. 18(1), 1 - 11 (2011)

8. Akram Ali, Wan A. Mior Othman, Cepar Ozel, Journal of Inequalities and Applications 2015, 291 (2015) Doi 10.1186/s13660-015-0802-5.

9. B. Najafi, N.H. Kashani, Turkish Journal of Mathematics 37, 1040 - 1047 (2013) http://journals.tubitak.gov.tr/ma th/

10. T. Tshikuna-Matamba, Math. Pannon. 23(2), 291-298 (2012)

11. K.L. Sai Prasad, International Mathematical Forum 4(16), 773 - 778 (2009)

12. A. Abu-Saleem A.R. Rustanov, Malaysian Journal of Mathematical Sciences 9(2), 187207 (2015)

13. A. Abu-Saleem, A.R. Rustanov, International Mathematical Forum 12(2), 87 - 95 (2017) https://doi.org/10.12988/imf.2017.611149

14. K. Erken, P. Dacko, C. Murathan, Mediterranean Journal of Mathematics 13(6), 4497 4507 (2016)

15. G.P. Singh, S.K. Srivastava, International Journal of Mathematics Research 8(1), 19-24 (2016)

16. S. Uddin, W.A.M. Othman, C. Ozel, Non-existence of warped product submanifolds of nearly Kenmotsu manifolds (2019) 
17. G. Ayar, M. Yildirim, Asian-European Journal of Mathematics 126, 2040002 (2019) DOI: $10.1142 / \mathrm{S} 1793557120400021$

18. A. Abu-Saleem, A.R. Rustanov, T.L. Melekhina, Chebyshevskii sbornik 20(2), 7 - 21 (2018)

19. A. Abu-Salem, A.R. Rustanov, Far East Journal of Mathematical Sciences (FJMS) 103(9), $1407-1432$ (2018) http://www.pphmj.com http://dx.doi.org/10.17654/MS103091407

20. A. Abu-Saleem, A.R. Rustanov, S.V. Kharitonov, Vladikavk. mat. zhurn. 20(3), 4-20 (2018) DOI: https://doi.org/10.23671/VNC.2018.3.17829

21. V.F. Kirichenko, A.R. Rustanov, Mathematical collection 193(8), 71-100 (2002) DOI: https://doi.org/10.4213/sm675

22. V.F. Kirichenko, Differential-geometric structures on manifolds. Second edition, supplemented (Printing House, Odessa, 2013)

23. Abu Saleem Ahmad, A.R. Rustanov, S.V. Kharitonov, Bulletin of Tomsk State University. Mathematics and Mechanics 66 (2020) DOI: 10.17223/19988621/66/1

24. V.F. Kirichenko, Dokl. RAS 380(5), 585-587 (2001)

25. V.F. Kirichenko, C.R. Acad. Sci. Paris. Sér. I. Math. 295(1), 673-676 (1982) 